\title{
Does seasonality of births in diabetes mellitus reflect pathogenetic differences?
}

\author{
Mikulecky $\mathrm{M}^{1}$, Rausova $\mathrm{Z}^{3}$, Dedik L4, Mojto $\mathrm{V}^{2}$ \\ Ist Internal Clinic, Faculty of Medicine, Comenius University, Bratislava, Slovakia. viliam.mojto@gmail.com
}

\begin{abstract}
OBJECTIVES: This study indicates that the seasonality of births of patients with DM1 and DM2 has occurred in their adolescence or adulthood.

BACKGROUND: Patients with Diabetes mellitus type 2 (DM2) with the maturity onset have different seasonal birth patterns from those with Diabetes mellitus type 1 (DM1) with the maturity onset, or DM1children.

METHODS: Monthly numbers of births of 81 and 236 children with DM1 and DM2, respectively, in adolescent or adult age, were adapted to different actual length of calendar months. The 12- and 6-month rhythm was tested using the cosinor regression with $95 \%$ confidence interval versus the hypothesis of null seasonality.

RESULTS: Regarding DM1 with maturity onset, annual and semiannual rhythm was significant in both genders, with the increase in birth numbers from November to January and decrease in March, April and August. In DM2, only female data displayed a significant annual rhythm, with an increase in birth from April to August and decrease from October to December.

CONCLUSION: The birth seasonality related to DM1 in adolescent or adult age appears to be reciprocal, compared to DM1 in childhood. For DM2, the seasonality of births was found only in females. The increase in female fecundity seems to be related to an increase in the risk of DM2 in female offspring. The outcomes could help in identifying environmental and endogenous factors related to seasonality cycle (Tab. 1, Fig. 1, Ref. 18). Text in PDF www.elis.sk. KEY WORDS: adulthood onset, birth seasonality, diabetes mellitus type 1, diabetes mellitus type 2 .
\end{abstract}

\section{Introduction}

The search for seasonal accumulation of births of some diseases is recently increasingly popular in the hope of finding factors responsible for triggering the initial pathologic process before its overt manifestation. This is particularly true with respect to childhood diabetes mellitus type 1 (DM1). Since 1951 there were found 31 titles in the databases of OLDMEDLINE and MEDLINE dealing with the latter but only 2 dealing, and 4 partly dealing with DM1 manifested in adolescence and/or adulthood. However, only one sole study about natality in adulthood diabetes mellitus type 2 (DM2) was found. Moreover, the results are not unequivocal. The pathogenetic process of DM1 can be considered to be a drama, sometimes as early as with pre- or perinatal exposition, i.e. years before the catastrophe of manifestation. In children populations with medium and high incidence of DM1, usually a summer peak in births is found (1). Mothers who become pregnant during vi-

${ }^{1}$ Ist Internal Clinic, Faculty of Medicine, Comenius University, Bratislava, Slovakia, ${ }^{2}$ IIIrd Internal Clinic, Faculty of Medicine, Comenius University, Bratislava, Slovakia, ${ }^{3}$ Pharmacobiochemical Laboratory of IIIrd Internal Clinic, Faculty of Medicine, Comenius University, Bratislava, Slovakia, and ${ }^{4}$ Institute of Automation, Measurement and Applied Informatics, Faculty of Mechanical Engineering, Slovak University of Technology, Bratislava, Slovakia

Address for correspondence: V. Mojto, IIIrd Internal clinic, Faculty of Medicine, Comenius University, Limbova 5, SK-833 05 Bratislava, Slovakia. Phone: +421.2.59542349 ral epidemics may transmit a virus damaging the pancreatic beta cells or protecting antiviral antibodies prenatally or perinatally to the fetus. Another interpretation takes into account the shorter breastfeeding of children born in summer (2).

Our study aims to search for births seasonality in patients with DM1 and DM2 manifested in their adolescence or adulthood.

\section{Hypotheses}

Both DM1 and DM2 have known genetic backgrounds. This knowledge is currently expanded towards new pathologic entities with important implications for effective treatment $(3,4,5,6)$. The source of a possible DM1 seasonality is suggested in seasonal cycles of environmental triggering factors such as viral infection. In DM2, internal hormonal cycles, also seasonally dependent, can participate in the manifestation of the disease. The seasonality aspects are usually studied for manifestations, more rarely from the view of births. However, it is possible that the pathogenetic process in DM1 starts in utero. It is very important to fully understand the facet of timing of the DM pathogenesis from its onset in order to be able to perform the potentially effective interventions.

\section{Materials and methods}

\section{Empirical data}

All the cases of diabetes adopted in adolescence or adulthood, available during the study design from the documentation of the III. Internal clinic, Faculty of Medicine, Comenius University in 


\section{1-504}

Bratislava, were taken into the study. The diagnoses of DM1 and DM2 have been established using the known criteria (e.g. Andreoli et al, 1993; p. 514, Table 71-3) (3). No patient with latent autoimmune diabetes in adults (LADA) has been encountered. The patients living in the Slovak capital Bratislava and its surroundings were recruited from a relatively homogeneous Caucasian population. The procedures were performed in accordance with ethical standards of the institutional research committee and with the 1964 Helsinki declaration and its later amendments, or with comparable ethical standards.

For DM1, a subgroup comprised of 6 adolescent males (according to the year of DM1 manifestation) aged 15-18 years, 24 young adults aged 19-29 years and 11 adults aged 30 and older at DM1 manifestation, as well as a subgroup comprised of 8 adolescent females, 23 young adults and 9 adults were studied. The age at the DM1 onset in the male subgroup was distributed normally while in females it was not (d'Agostino test of normality). The average age at DM1 onset (95\% confidence interval) was 25.4 years (23.4-27.5 years) in males and the median age was 24.5 years (21.0-27.0 years) in females. There is no significant onset age difference between both genders.

For DM 2, subgroups of 104 adult-onset males and 132 adultonset females were studied. The age at DM2 onset was distributed normally in either subgroup. The average age at DM2 onset was 54.5 years (52.3-56.6 years) in males and 59.7 years (57.9-61.6 years) in females. Thus, the age at the onset was statistically significantly higher in females compared to males. Accordingly, the age at the onset was statistically significantly higher for DM2 than that for DM1 in either gender group. Either mean age of onset with its $95 \%$ confidence intervals is in good agreement with that usually given for DM 1 (< 30 years) and DM 2 (> 40 years).

The general Slovak child population is represented by all children born between 1974 and 1997, i.e. 1,017,270 boys and 970,406 girls (6). The data were modified with respect to varying lengths months (between 28 and 31 days), divided by the monthly number of boys or girls from the general population, born in a comparable year span, transformed to moving averages, from three values each, and expressed as relative numbers with the total yearly sum equal to 1 . Thus, the value of probability to be born equal to $1 / 12$ is expected for each month according to null hypothesis. The resulting values of probability for each month were processed by cosinor regression (7), using original software (8). The presence of 12and 6-month rhythms was tested by evaluation of significance for the difference of amplitude from zero. The resulting significance was decreased by using three times lower degrees of freedom due to the use of the moving averages. The total approximating function was displayed graphically with its $95 \%$ confidence interval. Significant departures of probabilities from $1 / 12$, corresponding with the MESOR (midline estimating statistic of rhythm), are visible at separate months as non-overlapping of the MESOR line by the confidence interval.

\section{Results}

\section{Evaluation of the hypotheses and predictions}

Figure 1 shows the outcome for subjects of either gender with DM 1, manifested in adolescence or adult age, and for women with DM2, manifested in adult age. The male DM 2 patients were omitted as they did not show any significant birth seasonality.

Table 1 summarizes the significant monthly departures of births probability from the mean probability, equal 1/12, for separate subgroups of DM1and DM2 patients and for general population of Slovak children (6).

The DM1 data of children (1,186 patients, born in 1974-1997, manifested in 1989-1997) were taken from the studies of Mikulecký et a. $(9,10)$, while those for adults were taken from this study and from the study of Hummel et al (11) and Mikulecký (12). The analogical results for Crohn disease $(13,14)$ were added for comparison. Another useful comparison is related to the monthly excursions of the acute antiCX positivity on Coxsackie viruses in apparently healthy Slovak children (707 subjects) (10). Finally, the results for DM2 are those presented in the present study.

For DM1, the difference between our children $(9,10)$ and our adults is striking. It looks like shifting the months with significant elevation of DM1 births from children' spring - summer - early fall peaking towards the adults' late fall - early winter one. That

Tab. 1. Statistically significant elevations (+) or depressions (-) of monthly births' probability for separate samples and those of the anti-CX positivity on acute Coxsackie viruses' infections in apparently healthy Slovak children.

\begin{tabular}{|c|c|c|c|c|c|c|c|c|c|c|c|c|c|c|}
\hline & & Jan & Feb & Mar & Apr & May & Jun & Jul & Aug & Sep & Oct & Nov & Dec & $\mathrm{n}$ \\
\hline \multirow{5}{*}{ DM1 } & $\mathrm{cm}$ & $-r$ & - & & $+r$ & & & & & $+r$ & + & & $-r$ & 615 \\
\hline & $\mathrm{cf}$ & - & - & & $+\mathrm{r}$ & + & + & + & + & + & + & $-r$ & $-r$ & 571 \\
\hline & $\mathrm{am}$ & $+\mathrm{r}$ & & - & $-\mathrm{r}$ & & & & - & $-\mathrm{r}$ & & + & $+r$ & 41 \\
\hline & af & & - & - & $-r$ & & & & & & + & $+r$ & $+r$ & 40 \\
\hline & $\mathrm{a}^{*}$ & - & - & - & & & & & & + & + & + & & 1180 \\
\hline CD & $\mathrm{C}$ & $-\mathrm{r}$ & - & - & & & $+r$ & $+r$ & + & + & + & & $-\mathrm{r}$ & 66 \\
\hline AntiCX-pos & & - & - & - & - & - & - & + & + & + & + & + & - & 707 \\
\hline \multirow{2}{*}{ DM2 } & $\mathrm{am}$ & & & & & & & & & & & & & 104 \\
\hline & $\overline{\mathrm{af}}$ & & & & + & + & + & + & + & & - & - & - & 132 \\
\hline GP & & & + & + & + & + & + & + & & - & - & - & - & 1987676 \\
\hline
\end{tabular}

$\overline{\mathrm{DM}}$ - diabetes mellitus, c - children, a - adults, $\mathrm{a}^{*}$ - adults from Germany, $\mathrm{m}$ - males, $\mathrm{f}$ - females, $\mathrm{r}$ - reciprocity c versus a, CD - Crohn disease, GP - general population, $\mathrm{N}$ - sample size 


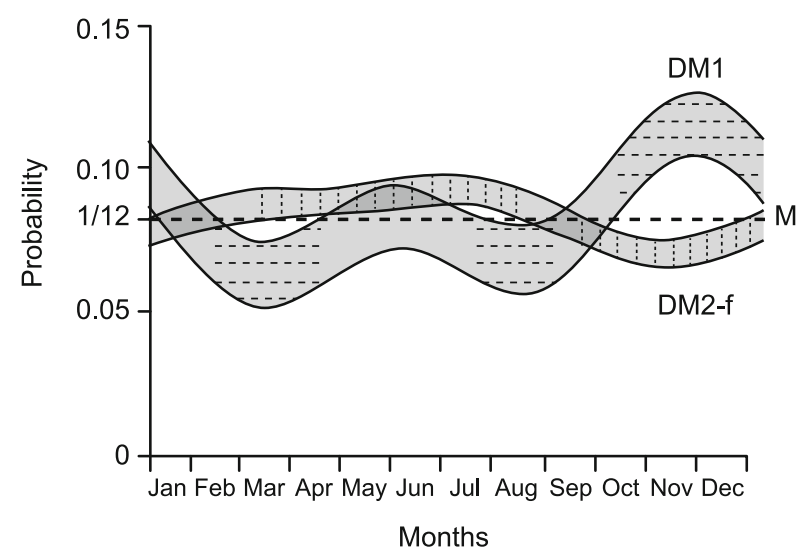

Fig. 1. Probability of being born in a given calendar month for subjects of either gender with Diabetes mellitus type 1 (DM1, horizontally shaded), and for females with Diabetes mellitus type 2 (DM2-f, vertically shaded) manifested in adolescent or adult age, as $95 \%$ confidence interval plotted versus months of calendar year. The MESOR (M) horizontal straight line (dashed) is also given. Statistically significant departures from the MESOR are shadowed.

could mean a shift in the relative (as to the time of birth) timing of a possible intrauterine infection, operated by the same, seasonally equally timed epidemiologic source, from a perinatal infection in childhood DM1 to prenatal one in adulthood DM1, where the births occur consequently later. The Coxsackie infections were peaking in the Slovak apparently healthy children each year in late summer and early fall (10). Their sharp peak in September is coincident with the elevations for childhood DM1 and Crohn disease (Tab. 1).

The most striking finding is the almost full identity of the birth pattern in the general population and in females with DM2, in the latter after being divided by the numbers from the whole population. The only reference for adulthood DM1 and DM2 from the study of Jongbloet et al (15) claims "excess of DM births in the first quarter of the year... and a deficiency in them during the last one". The latter statement is in agreement with our finding for DM2 in females but contrasts with our finding for DM1. In our adult DM1 patients, the excess of births in the first quarter is limited to January and males, while in our DM2 females, this excess is shifted by 3 months later, continuing through the second and partly through the third quarter of year. The close resemblance of the seasonal births' pattern in Slovak general population and in our female DM2 is intriguing. It seems like a maximal fecundity of females would be accompanied with increased tendency to develop DM2, nevertheless only in the female offspring.

It appears logical that for DM1, where an external cause is supposed, there is no difference in seasonality of births between genders, while for DM2 with a possible endogenous year cycle suggested by Jongbloet et al (15), only one gender - the female one - is involved in seasonal differences of the pathogenetic process.

\section{Discussion}

This study is not population-based. This should not pose a problem - it is hardly possible to suppose that the months of births of our patients could be biased, e.g. that patients born in October would prefer our hospital and those born in April did not. In other words, the months of birth in our tested subjects have to be considered strictly random. Very similar birth patterns were found for childhood Crohn disease (13) and for that adopted in adulthood (144).

The situation seems to be different in Germany where a distinct summer birth peaks of future DM1 adult patients (if not related to the general population!) was observed and considered similar to that in childhood DM1. The onset of the disease, however, in these "adults" was between 2 and 45 years, with the "mean age SD \pm $16.8 \pm 8$ years" - probably it should be read as "mean age \pm SD was $16.8 \pm 8$ years”. Accordingly, a substantial part of them (12) should be classified as having the childhood onset, not adulthood onset of diabetes. This feature has been obviously not understood appropriately by the respective authors (16). Their idea that possibly ,dividing the total population into different age groups will also change the results is hardly acceptable. Firstly, our Slovak population data cover a broad time span of 1974-1997 and the pattern of these data is stable. Secondly, it is not the actual age but the age of the onset of DM1 that has to be considered, and it has nothing to do with the births of general population. We think that our data not only support the "findings that the seasonality of birth of adult-onset patients with type 1 diabetes differs from that of the general population" (16) but could also support another finding, namely that adult-onset patients differ in birth seasonality also from childhood-onset adult DM 1 subjects. For the latter purpose, the separation of 1,180 data (11) with respect to the age of DM1 onset would be most desirable. Nevertheless, the births, after respecting the general population data, display also a shift towards November (Tab 1), probably due to partial presence of adulthood-onset DM1.

To arrive at valid conclusions concerning seasonality of births, the term "adult" diabetic patient has to be specified according to the age of manifestation of the original childhood onset and true adulthood-onset diabetes. In contrast to some other papers, the expression of results in our study is based on the inferential, inductive and not descriptive statistics, i.e. based on the estimates (e.g. confidence intervals), not basically on the tests. This approach was initiated in July 1905 by W. S. Gosset ("Student”) (17) and adopted relatively recently e.g. by British Medical Journal group (18).

\section{Conclusion}

The inferentially statistical analysis presented in an illustrative way in our study extends the knowledge about birth seasonality in patients with adulthood-onset DM1 and DM2, as documented by the search in the databases of OLDMEDLINE and MEDLINE. These conclusions should be guarded because of the relatively low sample sizes, particularly for DM1. Therefore, their validity can be limited to Slovakia and Central Europe. 


\section{1-504}

\section{References}

1. Laron Z. Interplay between heredity and environment in the recent explosion of the type 1 childhood diabetes mellitus. Am J Med Genet 2002; 115: 4-7.

2. Samuelsson U, Ludvigsson J. Seasonal variation of birth month and brestfeeding in children with diabetes mellitus. J Pediatr Endocrinol Metab 2001; 14: 43-46.

3. Andreoli TE, Bennett JC, Carpenter Ch CJ, Plum F, Smith LH, Jr. Cecil Essentials of Medicine. 3rd ed. Philadelphia, London, Toronto, Montreal, Sydney, Tokyo: WB Saunders Co., 1993: 921.

4. Karaman A, Bayram F, Gundogan K, Ozsan M, Karaman H, Kelestimur F. Prevalence of diabetes mellitus and glucose metabolism disorders in the first degree relatives of type 2 diabetic patients. Bratisl Lek Listy 2013; 113 (6): 361-367.

5. Kalninova J, Jakus V, Glejtkova M, Kuracka L, Sandorova E. Impact of glycemic control on advanced glycation and inflammation in overweight and obese patients with type 2 diabetes mellitus. Bratisl Lek Listy 2014; 115 (8): 457-468.

6. Kubáček L, Valach A. Time series analysis with periodic components. Computer programme. Bratislava: ComTel, 2005.

7. Mikulecký M. Seasonality of births in the whole Slovak population. Lek Obz 2003; 52: 363-365.

8. Bingham Ch, Arbogast B, Cornélissen GG, Lee JK, Halberg F. Inferential statistical methods for estimating and comparing cosinor parameters. Chronobiologia 1982; 9: 397-439.
9. Mikulecký M, Michalková D, Hlava P. Seasonality of births of Slovak IDDM children (Abstract). Diabetologia 1999; 42 (Suppl 1): A86/314.

10. Mikulecký M, Michalková D, Petrovičová A. Coxsackie infection and birth of future diabetic children: Year seasonality and secularity. J Ped Endocrinol Metabol 2000; 13: 523-527.

11. Hummel M, Lewy H, Ziegler AG, Ashkenazi I, Laron Z. Pattern of month of birth in diabetic and nondiabetic adults. Isr Med Assoc J 2002; 4: 74.

12. Mikulecký M. Birth seasonality in diabetic adults: reevaluation according to the age at disease onset. Letter. Israel Med Assoc J 2004; 6: 647-648.

13. Mikulecký M, Čierna I. Seasonality of births and childhood inflammatory bowel disease. Wien Klin Wschr 2005; 117: 554-557.

14. Mikulecký M, Rovenský J, Kmečová Z. Chronobiological aspects of adult onset Crohn disease. Wien Klin Wschr 2005; 117: 423-428.

15. Jongbloet PH, Van Soestbergen M, Van der Veen EA. Month - of birth distribution of diabetics and ovopathy: a new aetiological view. Diabet Res 1988; 9: 51-58.

16. Laron Z, Lewy H. Birth seasonality in diabetic adults: reevaluation according to the age at disease onset. Letter. Israel Med Assoc J 2004; 6: 648.

17. Fedor-Freybergh PG, Mikulecký M. From the descriptive towards inferential statistics. Hundred years since conception of the Student' s tdistribution. Neuro Endocrinol Lett 2005; 26: 167-171.

18. Gardner MJ, Altman DG. Confidence intervals rather than $P$ values: estimation rather than hypothesis testing. Br Med J 1986; 292: 746-750.

Received March 2, 2016. Accepted March 21, 2016. 